\title{
Classification of leakage detections acquired by airborne thermography of district heating networks
}

\author{
Amanda Berg and Jörgen Ahlberg
}

Linköping University Post Print

Tweet

N.B.: When citing this work, cite the original article.

C2014 IEEE. Personal use of this material is permitted. However, permission to reprint/republish this material for advertising or promotional purposes or for creating new collective works for resale or redistribution to servers or lists, or to reuse any copyrighted component of this work in other works must be obtained from the IEEE.

Amanda Berg and Jörgen Ahlberg, Classification of leakage detections acquired by airborne thermography of district heating networks, 2014, Pattern Recognition in Remote Sensing. http://dx.doi.org/10.1109/PRRS.2014.6914288

Postprint available at: Linköping University Electronic Press http://urn.kb.se/resolve?urn=urn:nbn:se:liu:diva-110046 


\title{
Classification of leakage detections acquired by airborne thermography of district heating networks
}

\author{
Amanda Berg*† and Jörgen Ahlberg*† \\ *Dept. of Electrical Engineering \\ Linköping University \\ SE-581 83 Linköping, Sweden \\ ${ }^{\dagger}$ Termisk Systemteknik AB \\ Diskettgatan 11B \\ SE-583 35 Linköping, Sweden \\ E-mail: \{amanda.berg, jorgen.ahlberg\}@liu.se
}

\begin{abstract}
We address the problem of reducing the number of false alarms among automatically detected leakages in district heating networks. The leakages are detected in images captured by an airborne thermal camera, and each detection corresponds to an image region with abnormally high temperature. This approach yields a significant number of false positives, and we propose to reduce this number in two steps. First, we use a building segmentation scheme in order to remove detections on buildings. Second, we extract features from the detections and use a Random forest classifier on the remaining detections. We provide extensive experimental analysis on real-world data, showing that this post-processing step significantly improves the usefulness of the system.
\end{abstract}

\section{INTRODUCTION}

District heating networks distribute heat through underground pipes carrying hot water or steam from a central power plant. Heat or water leakages due to bad insulation or cracks are common problems. The pipes degenerate with time [3] and in some cities the pipes have been used for several decades. Loss of media (water/steam) or energy is expensive and has negative impact on the environment [4]. It is therefore of great interest to the network owners to find methods to detect and localize the leakages. The fact that the pipes are placed underground increases the need, as it is very expensive to dig in the wrong place. Moreover, major leakages (in the order of $50-150 \mathrm{~m}^{3}$ of media per day) may cause the ground to collapse due to erosion, whereby large amounts of water at boiling temperature are exposed.

\section{A. Related work}

Methods for monitoring of district heating networks have been developed over the years, for example methods based on frequency response or change in electrical impedance for a thread installed inside the pipe insulation. It is also common to measure the flow of water or steam in the inlet and outlet. If it differs, there is a leakage somewhere along the pipe. Such methods can be used to detect the presence of a leakage and sometimes its approximate location (which pipe segment). However, the exact location, needed for digging, is not revealed.

Methods for large-scale monitoring by aerial thermography, that is remote sensing from an aircraft using a thermal camera, have been investigated by Ljungberg et. al in the 80's (e.g. [5]) and Axelsson [6]. Also, ground-based thermography has been investigated using hand-held cameras [7], [8]. Compared to aerial thermography, this has several drawbacks, such as restricted access to many areas of interest and less scaleability.

The first system with automatic image analysis was presented by Friman et al. [1]. The system uses anomaly detection in order to detect abnormally warm areas along the pipes. However, the problem is the large number of false alarms since there are many areas that, for one reason or another, are warmer than the surroundings. To reduce the number of false alarms, buildings are segmented in order to avoid detections due to, e.g., chimneys when the pipes pass under buildings.

\section{B. Contribution}

The main contribution of the present work is to characterize the detections obtained using the method presented by Friman et. al [1], and then to classify them as real leakages or false detections. The two subproblems of feature selection and classification methods are both addressed. Moreover, we propose to do the building segmentation differently compared to [1].

\section{Outline}

The outline of this paper is as follows. In Section II, the acquisition of data and the resulting data sets are described. Section III describes our method and how it adds on to existing methods. Experiment and results are described in Section IV, and, finally, Section V contains our conclusions.

\section{DATA ACQUiSITION AND LEAKAGE DETECTION}

This section briefly describes the data acquisition process, pre-processing of the thermal images and the employed detection method. This corresponds to the scheme described in [1], but is included here in order to make the paper self-contained.

\section{A. Image data}

The thermal images are acquired from an aircraft. GPS and IMU are used to record the position and orientation of the aircraft in order to facilitate georeferencing. The imagery is georeferenced using semi-automatic commercial off-the-self software. 
TABLE I

NUMBER OF SAMPLES FOR EACH LAYER AND CLASS

\begin{tabular}{lcccccc}
\hline Layer no. & 1 & 2 & 3 & 4 & 5 & 6 \\
\hline Threshold & $0.05 \%$ & $0.1 \%$ & $0.5 \%$ & $1 \%$ & $3 \%$ & $5 \%$ \\
Media/Energy & 34 & 39 & 71 & 89 & 99 & 80 \\
False & 71 & 75 & 148 & 237 & 294 & 348 \\
\hline
\end{tabular}

The thermal camera is a cooled mid-wave infrared FLIR SC7000 Titanium with a resolution of $640 \times 512$ pixels and a field of view of $11^{\circ}$. At an altitude of $800 \mathrm{~m}$, this yields a pixel footprint of $25 \times 25 \mathrm{~cm}$.

In order to minimize the number of false detections, data collection is mainly done during the night or at dawn during spring or autumn. At this time, neither vegetation or snow is blocking the view, the effect from sun heating is minimal and the streets are not covered with cars blocking the view [9].

\section{B. GIS data}

The network owner provides pipe location information in the form of vector maps. This information is projected on top of the georectified images creating a rasterized pipe mask for each image. The mask is then used to limit the search for unnaturally high temperatures to areas above the pipes only.

\section{Detections}

A detection is in this context an area with a certain shape and location pointed out as abnormally warm. That is, it is an extended object, not just a coordinate. In order to extract the detections from the images, we use the anomaly based method described in [1]. Statistics of the ground temperature inside the pipe mask are calculated from all images within one flight and the most deviating pixels above certain thresholds in the high end of the distribution (i.e. the "warmest pixels") are marked as detections. The percentage thresholds are; $0.05 \%$, $0.1 \%, 0.5 \%, 1 \%, 3 \%$ and $5 \%$, resulting in six different layers of detections.

\section{Ground truth data}

Acquisition campaigns during the last couple of years have resulted in thousands of thermal images from 17 Scandinavian towns and cities. Three of the most recent acquisitions were selected for this study. The selection was based on the fact that the customers for these flights could provide ground truth, i.e., information about which detections had been investigated further and proven to be real (or false) leakages.

Detections from these cities have been manually labeled as media leakages, energy leakages, or false detections. To do this, interviews with the network owners have been conducted several months after the detection results have been delivered, that is, when the true status of the pipes in many cases have been investigated (by digging). Also, many detections can be pointed out as false by the network owners or by ourselves.

In total, we have 1585 labeled detections, of which 1173 are false. The number of samples for each class and layer can be seen in Table I.

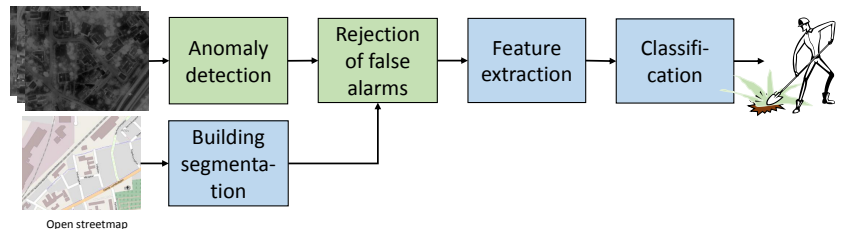

Fig. 1. The proposed approach.

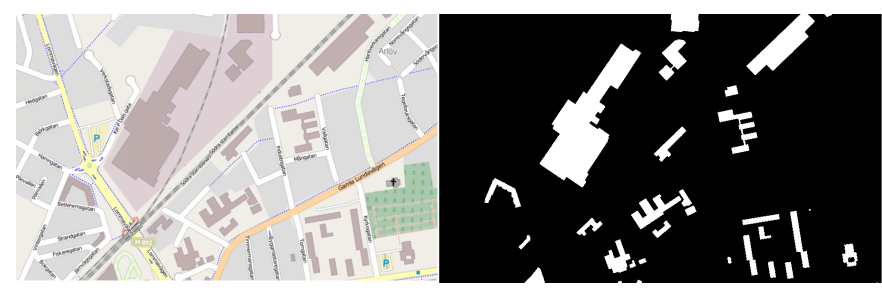

Fig. 2. An example of a building mask (right) generated from the colour raster map OpenStreetMap (left).

\section{METHOD}

Our approach is illustrated in Figure 1. The green boxes are identical to the scheme by Friman et al., while the blue ones are added or modified. Below we will describe the proposed building segmentation scheme, and then the feature extraction and classification.

\section{A. Removing false detections using building segmentation}

A common source for false alarms are detections of objects, e.g., chimneys and atriums, at rooftops with unnaturally high temperatures. These false alarms appear because the pipes sometimes pass beneath buildings. Since we know that real leakages of the district heating network never can appear at rooftops, information on building locations can be used to remove false detections. Friman et al. implemented a building segmentation scheme based on the watershed transform and AdaBoost classification in order to automatically extract building information from the thermal imagery.

The proposed building segmentation method is based on OpenStreetMap ${ }^{1}$. A binary building mask is generated using a simple color segmentation scheme, since buildings in these images have a specific set of colors; an example can be seen in Figure 2. By thresholding the three RGB channels, each pixel is classified as belonging to a building or not. The thermal images as well as the OpenStreetMap-images are stored in GeoTIFF format with world coordinate information related to each pixel, information that is used for image registration.

\section{B. Removing false detections using a classifier}

The basic assumption for our approach is that distinguishing features of the different types of detections do exist. Such features could be collected from the imagery or from the detections themselves (shape descriptors). The labeled examples should be studied to find an initial set of discriminating features which is later refined. The extracted features are then used to evaluate a number of classifiers. Below, we describe

\footnotetext{
${ }^{1}$ http://openstreetmap.org
} 
the selection of features and classifiers to use in the final system.

\section{Features}

Features were found by studying the labeled samples. The initial selection consisted of 18 scalar features based on (thermal) intensity distribution within the detection, shape and propagation of the detection, and proximity information. Feature selection, described further in section IV-B, was performed using the Mahalanobis distance.

\section{Classifiers}

Two linear and three nonlinear classifiers were chosen for evaluation. The linear ones are Linear Discriminant Analysis (LDA) [10] and Linear Support Vector Machines [11]. These were mainly included in the experiments to exclude linear methods from further evaluation. Three different nonlinear classifiers were evaluated; the Radial Basis Function Support Vector Machine [11], AdaBoost [12], and Random forest [13]. We used the implementations from [15].

For each nonlinear classifier, the choice of parameters was evaluated and chosen iteratively starting at the default value and moving in the direction of steepest descent of false positive rate until convergence. The chosen parameters and the default values can be seen below (chosen/default value).

- RBF-SVM: $\sigma=140 / 1$

- Random forest: No. of decision trees: 120 / 50

- AdaBoost: No. of weak classifiers: 192 / 100

Apart from these parameters, the weak classifier used in AdaBoost was the decision stump and the number of random features used for node splitting in Random forest was one.

\section{E. Evaluation and selection methodology}

There are three main types of detections; media, energy and false detections. Since both media and energy detections are interesting for the network owner, we chose to combine the media and energy samples into one class, hereafter called true detections. Furthermore, incorrect classification of one of these classes as the other one is not as critical as incorrect classification between media/false and energy/false.

As mentioned before, the cost for classifying a true detection as a false one is much higher than classifying a false detection as a true one. Therefore, the true positive rate was set to a specific value, namely $99 \%$, and the error measurement used for the evaluation was the false positive rate.

\section{EXPERIMENTAL RESULTS}

\section{A. Building segmentation}

Two building segmentation schemes were evaluated. The scheme proposed by Friman et al. [1] using the thermal imagery, and the scheme using OpenStreetMap. It was observed that both schemes sometimes result in errors, but the errors were of different character. The method in [1] sometimes suffer from non-building areas being classified as buildings, which could potentially make true detections (i.e.,

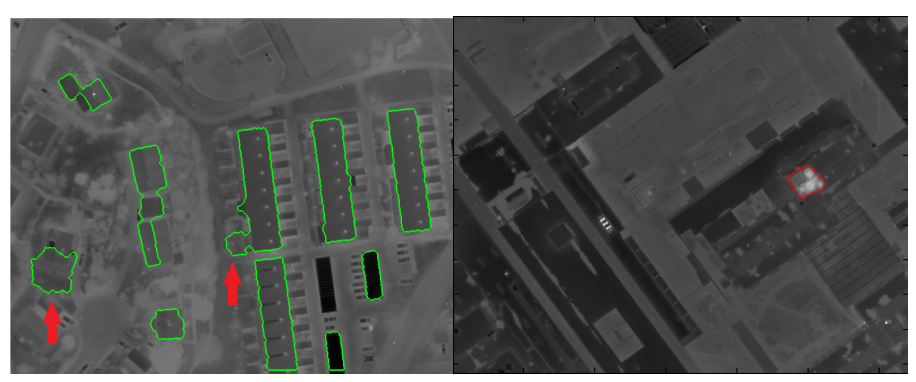

(a)

(b)

Fig. 3. (a) shows an example of the performance of the building segmentation method proposed by Friman et al. [1]. The green boundaries indicate the areas that have been classified as buildings. The arrows show the problem areas. Unacceptable classification of ground as buildings which could lead to missed real leakages. (b) shows instead an example of a successfully classified false detection, indicated with red boundaries, when the OpenStreetMap-based scheme is used.

real leakages) be discarded. In contrast, the OpenStreetMapbased scheme sometimes suffer from missing buildings, which might result in false detections classified as true detections. As mentioned, the cost for the latter is much lower, thus we selected the OpenStreetMap-based scheme. Examples of the performance of both methods can be seen in Figure 3.

Using the OpenStreetMap-based scheme described, removing all detections which lie $100 \%$ on top of a building, $19 \%$ of the false detections in the data set could be removed without removing any of the energy or media samples.

\section{B. Feature selection}

Feature selection was done by calculating the Mahalanobis distance between class means for each feature. As the Mahalanobis distance increases and approaches infinity, the probability of error decreases. Therefore, the Mahalanobis distance is a common choice for measuring the "goodness" of a feature [14].

The final set of features are the eight features for which the ground truth samples had the largest average Mahalanobis distance. The features are listed and described in Table II.

\section{Classifier selection}

Each classifier was evaluated using 10-fold cross-validation and the 8 features described above. The classifiers were trained and tested on each layer individually.

The averaged results over all layers are shown, for each individual classifier, in Figure 4. Apparently, the Random forest classifier outperforms the others. Combining the results from the building based rejection and the classification, the weighted (with respect to number of false samples in each layer) averaged false positive rate across layers is $58 \%$.

\section{Classification and detection layers}

As mentioned, the detection method results in several layers of detections, where the first layer contains the "worst" detections only (the ones with the most anomalous temperatures). In the previous sections, training and classification were done separately for each layer. We have investigated whether these 
TABLE II

FEATURES USED FOR CLASSIFICATION

\begin{tabular}{ll}
\hline Feature & Description \\
\hline Median intensity & Median intensity within the detection. \\
Standard deviation & $\begin{array}{l}\text { Standard deviation of the intensity within } \\
\text { the detection. } \\
\text { Ratio of detection area inside heat pipe } \\
\text { mask. } \\
\text { area where } d \text { is the number of erosions } \\
\text { needed to make the detection disappear. } \\
\text { Elongatedness }\end{array}$ \\
Concentricity & $\begin{array}{l}\text { Measurement of how central the maximum } \\
\text { intensity value is within the detection. }\end{array}$ \\
Connected components & $\begin{array}{l}\text { Number of other detections which lie within } \\
\text { a certain radius from the detection. }\end{array}$ \\
Border average & $\begin{array}{l}\text { Mean intensity within an area around the } \\
\text { detection. } \\
\text { Distance from maximum intensity value to } \\
\text { the wall of the closest building. }\end{array}$ \\
\hline
\end{tabular}

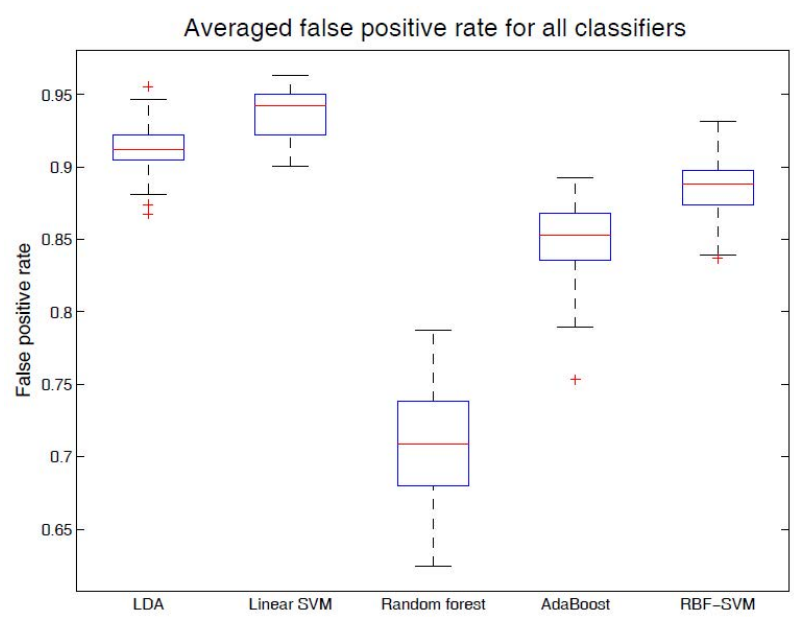

Fig. 4. Averaged false positive rate for all evaluated classifiers and thresholds. The red line shows the median, the blue box the 25th and 75th percentile and the whiskers mark the most extreme samples not considered as outliers. Outliers are plotted as red crosses.

classifiers can be combined, since (i) if a detection is present in several layers, adding information from the other layers might improve robustness, and, (ii) the differences in classification output from the different layers indicate the robustness of the classifiers.

1) Voting: Since detections corresponding to the same area often are present in several layers, the possibility of improving the result by voting between layers was investigated. However, in all the available data, no occasion of dissimilar labeling by different threshold classifiers was observed. Thus, the classifier is apparently robust to changing temperature thresholds.

2) Layer invariant classification: For the evaluation of layer invariant classification, all the 1585 detections from the six layers were combined to form one large data set.

Since the Random forest classifier have shown to achieve the best general performance for all percentage thresholds, it was chosen to be the classifier also for the threshold invariant classification. Combining the results from the building based rejection and the classification, the false positive rate was $42 \%$. Thus, to our surprise, this combined classifier gives better performance (on average) than a set of specialized classifiers. However, the results for the set of specialized classifiers is somewhat unreliable for layer 1 and 2 due to the small number of samples.

\section{CONCLUSION}

We have presented an improvement to a previously published method for finding leakages in district heating networks by classifying its resulting detections using trained classifiers. We have evaluated various features and five different classifiers. The classifier that generally produced the smallest false positive rate while maintaining a true positive rate of $99 \%$ is a Random forest classifier with 120 trees, an average tree depth of 10 and splitting at nodes based on one randomly selected feature. The classification of different layers of detections have been investigated, and the results indicate that the classification is consistent over layers

We have also shown that a pre-processing step for building extraction using publicly available GIS-data is preferable compared to the previously used method, not due its superior performance in general, but due to the different type of errors produced by the two methods.

In the end, we were able to achieve a false positive rate of $42 \%$, that is, we can discard $58 \%$ of the detections given by the previous system, thus significantly enhancing the usability.

\section{REFERENCES}

[1] O. Friman et al., "Methods for large-scale monitoring of district heating systems using airborne thermography," IEEE Trans. Geoscience and Remote Sensing, 52(8):5175-5182, 2014.

[2] A. Poredos and A. Kitanonovski, "District heating and cooling for efficient energy supply," in Proc. Int. Conf. Electrical and Control Engineering, 2011, pp. 5238-5241.

[3] M. Olsson, "Long-term thermal performance of polyurethane-insulated district heating pipes," Ph.D. thesis, Chalmers Univ. of Techn., 2001

[4] M. Fröling, "Environmental and thermal performance of district heating pipes," Ph.D. thesis, Chalmers Univ. of Techn., 2002.

[5] S.-A. Ljungberg, "Aerial thermography - a tool for detecting heat losses and defective insulation in building attics and distric heating networks," in Proc. SPIE Thermosense IX, 1987, pp. 257-265.

[6] S. R. J. Axelsson, "Thermal modeling for the estimation of energy losses from municipal heating networks using infrared thermography," IEEE Trans. Geoscience and Remote Sensing, 26(5):686-692, 1988.

[7] B. Bøhm and M. Borgström, "A comparison of different methods for in-situ determination of heat losses from district heating pipes," Dept. of Energy Engineering, Technical Univ. of Denmark, 1996.

[8] H. Zinko et al., "Quantitative heat loss determination by means of infrared thermography - the TX model,” Int. Energy Agency, 1996.

[9] S. Sjökvist at al., "Kvantifiering av värmeläckage genom flygburen IRteknik - en förstudie," Fjärrsyn, Rep. 2012:17, 2012

[10] R. O. Duda et al., Pattern Classification, John Wiley \& Sons, 2001.

[11] T. Hastie, R. Tibshirani, and J. Friedman, The elements of statistical learning, 2nd ed. Springer, 2008.

[12] C. M. Bishop, Pattern Recognition and Machine Learning, Springer, 2006.

[13] L. Breiman, "Random forests," Machine learning, 45(1):5-32, 2001.

[14] A. Jain and D. Zongker, "Feature selection: Evaluation, application, and small sample performance," IEEE Trans. Pattern Anal. Mach. Intell., 19(2):153-158, 1997.

[15] R. P. W. Duin et al., "PR-Tools4.1: A Matlab Toolbox for Pattern Recognition," Delft University of Technology, 2007, http://www.prtools.org. 